\title{
Achievable Degrees of Freedom on $K$-user MIMO Multi-way Relay Channel with Common and Private Messages
}

\author{
Mohamed Salah ${ }^{\dagger}$, Amr El-keyi ${ }^{\dagger}$, Yahya Mohasseb ${ }^{\dagger *}$ and Mohammed Nafie ${ }^{\ddagger}$ \\ ${ }^{\dagger}$ Wireless Intelligent Networks Center (WINC), Nile University, Cairo, Egypt \\ *Department of Communications, The Military Technical College, Cairo, Egypt \\ ${ }_{\ddagger}$ EECE Dept., Faculty of Engineering, Cairo University, Giza, Egypt \\ Email: $\{m . s a l a h @ n i l e u . e d u . e g, ~ a e l k e y i @ n i l e u n i v e r s i t y . e d u . e g, ~\{m n a f i e, m o h a s s e b\} @ i e e e . o r g\}$
}

\begin{abstract}
This paper investigates the achievable total degrees of freedom (DoF) of the MIMO multi-way relay channel that consists of $K$ users, where each user is equipped with $M$ antennas, and a decode-and-forward relay equipped with $N$ antennas. In this channel, each user wants to convey $K-1$ private messages to the other users in addition to a common message to all of them. Due to the absence of direct links between the users, communication occurs through the relay in two phases: a multiple access channel (MAC) phase and a broadcast (BC) phase. We derive cut-set bounds on the total DoF of the network, and show that the network has DoF less than or equal to $K \min \{N, M\}$. Achievability of the upper bound is shown by using signal space alignment for network coding in the MAC phase, and zeroforcing precoding in the $\mathrm{BC}$ phase. We show that introducing the common messages besides the private messages leads to achieving higher total DoF than using the private messages only.
\end{abstract}

\section{INTRODUCTION}

The use of relays has drawn much research interest in wireless networks. This is mainly due to its ability to increase the overall throughput, improve the energy efficiency, and extend the coverage in the case of power limited terminals [1]. However, the main focus so far is on the mutli-way communication where an additional node acting as a relay is supporting the exchange of information between a group of users. Since characterizing the capacity of the MIMO relay networks is too complex, a lot of work was done to study such networks using an alternative metric, which is the degrees of freedom $(\mathrm{DoF})$.

Recently, Lee et. al. studied the DoF of the symmetric 3-user MIMO Y channel in [2]. In this channel, each user equipped with $M$ antennas aims to deliver two private messages to the other two users via a decode and forward relay equipped with $N$ antennas. They showed that if $N \geq 3 M / 2$, then the cut-set bound given by $3 M \mathrm{DoF}$ is achievable. However, the novel contribution is not in achieving the DoF upper bound of the network but in their new idea of alignment for network coding. They exploit the concept of interference

This paper was made possible by a grant from the Egyptian National Telecommunications Regulatory Authority. The statements made herein are solely responsibility of the authors. alignment and Physical Layer Network Coding (PLNC) to propose a Signal Space Alignment technique for Network Coding (SSA-NC). The main idea of SSA is that each pair of users who want to exchange messages cooperatively designs beamforming matrices such that the bidirectional vectors of each pair of users can be aligned at the relay in the same subspace. Then, the relay can simply decodes and forwards the linear combination of every pairwise vectors. Finally, each user can decode desired vectors by subtracting its side information from the network coded received vectors. Thus, the phrase of SSA-NC means signal alignment at the relay and network coding at users for decoding.

Based on the work of [2], total DoF of different scenarios has been characterized in [3]-[7]. Particularly, the authors in [3] investigated the total DoF of the 3-user asymmetric MIMO Y channel. They showed that the general MIMO $\mathrm{Y}$ channel with $M_{1} \geq M_{2} \geq M_{3}$ antennas at user 1, 2, and 3, respectively, and with $N$ antennas at the relay, has $\min \left\{2 M_{2}+2 M_{3}, M_{1}+M_{2}+M_{3}, 2 N\right\}$ DoF. They derived an outer bound on the DoF using cut-set bounds and genieaided bounds. Then, the work in [4] extended the channel model in [2] to the case of $K$ users, where the $j$-th user with $M_{j}$ antennas sends $K-1$ independent messages via the relay equipped with $N$ antennas and each message achieves $d$ DoF. They proved the achievability of total $\mathrm{DoF}=d K(K-1)$ using SSA-NC. In [5], the authors studied the DoF of the 4-user relay network with two clusters of users where each cluster contains two users. Each user wants to exchange messages with the other user in the same cluster. They achieved a total DoF of $2 \min \{2 M, N\}$ using time division multiple access and SSA-NC, where $M$ and $N$ are the number of antennas at each user and the relay, respectively. Then, they extended this work to the case of $L$-clusters, $K$-user MIMO multi-way Relay Channel (mRC) with no direct links, where users in each cluster wish to exchange messages within the cluster [6]. Finally, the authors of [7] generalized the work of [3][6] to the case where each user can arbitrarily select one or more partners to conduct independent information exchange. They showed that the upper bound on the total DoF of 
$\min \left\{\sum_{i=1}^{K} M_{i}, 2 \sum_{i=2}^{K} M_{i}, 2 N\right\}$ is tight under the antenna configuration $N \geq \max \left\{\sum_{i=1}^{K} M_{i}-M_{s}-M_{t}+d_{s, t} \mid \forall s, t\right\}$, where $M_{i}$ and $N$ are the number of antennas at node $i$ and the relay, respectively, and $d_{s, t}$ denotes the DoF of the message exchanged between nodes $s$ and $t$.

In this paper, we consider the network information flow problem for a $K$-user Gaussian MIMO $\mathrm{mRC}$ and analyze its DoF. In this channel, all the $K$ users are equipped with $M$ antennas and the relay is equipped with $N$ antennas. However, unlike most of the prior work where the users were assumed to exchange private messages only [2]-[7], we consider both common and private messages. The $j$-th user intends to convey $K$ messages to the other users by the help of an intermediate relay; $K-1$ private messages to the other users in addition to a common message to all of them, where $j \in\{1, \cdots, K\}$. We first derive cut-set bound on the total DoF, and show that the network has $K \min \{N, M\}$ DoF. By combining PLNC and SSA, we then propose a novel and systematic way of beamforming design at the users and at the relay to align signals, and consequently, we can efficiently implement PLNC. Additionally, we show that our proposed scheme can achieve this upper bound.

The rest of this paper is organized as follows: In Section [I]. we describe our network and the main assumptions. Next, we derive cut-set bounds on the total DoF in Section [II] Then, we present the proposed achievable scheme in Section IV] Finally, our conclusions are stated in Section V

\section{Notation}

In this work, boldface uppercase letters denote matrices and boldface lowercase letters are used for vectors. $\mathbb{C}$ denotes the complex space. For any general matrix $\boldsymbol{G}, \boldsymbol{G}^{H}, \boldsymbol{G}^{\dagger}, \mathcal{S}\{\boldsymbol{G}\}$ and $\mathcal{N}\{\boldsymbol{G}\}$ denote the Hermitian transpose, the pseudo inverse, the span of the column vectors, and the null space of $\boldsymbol{G}$, respectively.

\section{System Model}

\section{A. Channel Model}

The system model for $K$-user MIMO $\mathrm{mRC}$ is shown in Fig. 11 In this channel, $K$ users, each equipped with $M$ antennas, exchange messages in a pairwise manner with the help of an intermediate $N$ - antennas relay node. Specifically, user $j$ wants to send $K$ messages; $K-1$ private messages $\mathbf{m}_{j i}$ to user $i$ in addition to a common message $\mathbf{m}_{j c}$ to the remaining $K-1$ users. Also, the $j$-th user intends to decode all the other users' messages; $K-1$ private messages $\hat{\mathbf{m}}_{i j}$ in addition to $K-1$ common messages $\hat{\mathbf{m}}_{i c}$, where $i, j \in\{1, \cdots, K\}, i \neq j$. It is assumed that the users can communicate only through the relay and no direct links exist between any pairs of users. Additionally, we assume that the channel between each user and the relay is reciprocal. The transmission takes place over two phases: MAC and BC. In the MAC phase, each user transmits its messages to the relay.

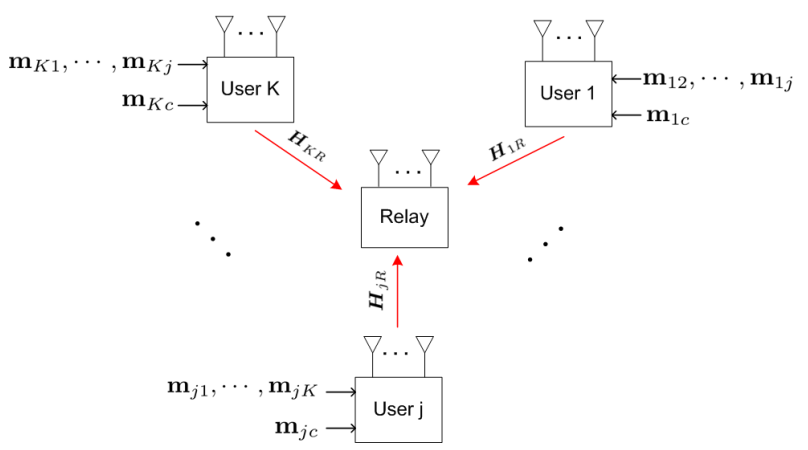

(a)

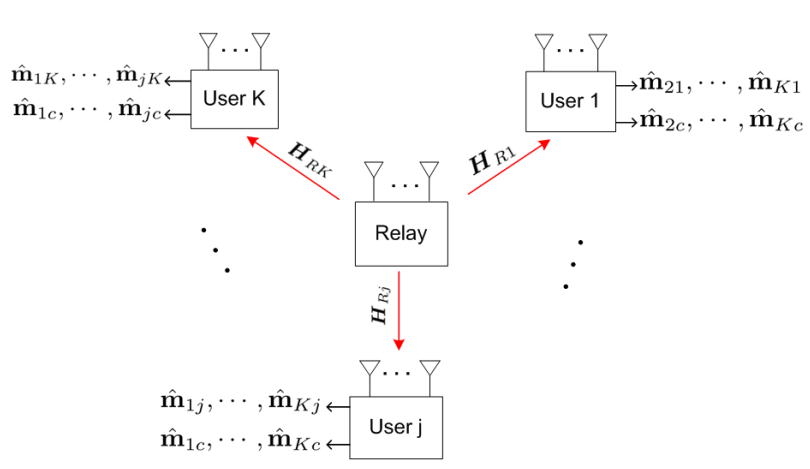

(b)

Fig. 1: System Model of $K$ user MIMO mRC: $\{$ a) MAC phase, (b) Broadcast phase \}

The received signal at the relay is given by

$$
\mathbf{y}_{r}=\sum_{j=1}^{K} \boldsymbol{H}_{j R} \mathbf{x}_{j}+\mathbf{z}_{r}
$$

where $\mathbf{y}_{r}$ and $\mathbf{z}_{r}$ denote the $N \times 1$ received signal vector and additive white Gaussian noise (AWGN) vector with unit variance at the relay, respectively. $\mathbf{x}_{j}$ is an $M \times 1$ vector representing the transmitted signal from user $j . \boldsymbol{H}_{j R}$ is the $N \times M$ channel matrix from user $j$ to the relay, where the entries of the channel matrices $\boldsymbol{H}_{j R}$, for $j \in\{1, \cdots, K\}$, are independently and identically distributed zero mean complex Gaussian random variables with unit variance. Thus, we guarantee that each of the channel matrices is full rank almost surely. In the $\mathrm{BC}$ phase, the received signal at user $j$ from the relay is given by

$$
\mathbf{y}_{j}=\boldsymbol{H}_{R j} \mathbf{x}_{r}+\mathbf{z}_{j}
$$

where $\mathbf{y}_{j}$ and $\mathbf{z}_{j}$ are the $M \times 1$ received vector and AWGN vector at the $j$-th user, respectively. $\mathbf{x}_{r}$ is an $N \times 1$ vector representing the transmitted signal vector from the relay. Finally, $\boldsymbol{H}_{R j}$ denote the $M \times N$ channel matrix from the relay to user $j$. Throughout this paper, we assume that perfect channel state information is available at all users and at the relay in both phases. Also, we assume that all the users have 
the same power constraint. Moreover, all nodes are assumed to operate in full-duplex mode. ${ }^{1}$

\section{B. Degrees of freedom}

In the absence of precise capacity characterizations, researchers have pursued asymptotic and/or approximate capacity characterizations. One of the most important capacity characterizations is the DoF of the network [8]-[10]. The DoF metric is primarily concerned with the limit where the total transmit power approaches infinity, while the values of channel coefficients and the local noise power remain unchanged. Thus, if we denote by $C(P)$ the sum capacity with the total transmit power $P$, then the DoF metric $\eta$ is defined as

$$
\eta=\lim _{P \rightarrow \infty} \frac{C(P)}{\log (P)}
$$

In other words, we can consider the DoF as the number of signaling dimensions, where 1 signal dimension corresponds to one interference-free AWGN channel with SNR increasing proportionately with $P$ as $P$ approaches infinity. Let $d_{i j}$ denote the number of interference-free streams sent from user $i$ to user $j$. Also, we define $d_{j c}$ as the number of interferencefree streams sent from user $j$ to the remaining $K-1$ users. The total DoF of the MIMO mRC with private and common messages is given by the total number of interference-free streams sent through the network. As a result, the total DoF $D_{t}$ is equal to

$$
D_{t}=\sum_{j=1}^{K} \sum_{i=1, i \neq j}^{K} d_{j i}+(K-1) \sum_{j=1}^{K} d_{j c}
$$

Notice that in the above equation the contribution of the common messages to the total DoF is weighted by a factor $K-1$ as each common message is directed to $K-1$ users.

\section{UPPER BOUNDS ON THE DEGREES OF FREEDOM}

In this section, we provide upper bounds on the total DoF of the Gaussian MIMO mRC with common and private messages. One way to obtain upper bounds for the $K$-user MIMO mRC is by using the cut-set bounds. In [2], Lee $e t$ al. derived cut set bounds for the Gaussian MIMO Y channel with equal number of antennas at all users, where the users exchange private messages only. We introduce the common messages to the network and generalize these bounds for arbitrary number of users. Then, using the cut set-bounds, we can obtain the DoF bounds. The main result of this section is stated in the following theorem.

Theorem 1. The total number of DoF of a Gaussian MIMO $m R C$ is given by

$$
D_{t}=K \min \{N, M\}
$$

Proof:

\footnotetext{
${ }^{1}$ All the DoF results can be applied to the half-duplex case with only a multiplicative factor of $\frac{1}{2}$
}

Let us label the set of nodes in the network by $\mathcal{P} \triangleq$ $\left\{U_{1}, \cdots, U_{K}, R\right\}$, where $U_{j}$ denotes user $j$, for $j \in$ $\{1, \cdots, K\}$, and $R$ denotes the relay. Moreover, we define the two sub-sets $\mathcal{T}$ and $\mathcal{T}^{c}$ as the transmitting and receiving nodes, respectively, where $\mathcal{T}$ and $\mathcal{T}^{c} \in \mathcal{P}$. By considering only the messages intended to $U_{1}$ from $\left\{U_{2}, \cdots, U_{K}\right\}$ and writing the cut in the MAC phase where $\mathcal{T}=\left\{U_{2}, \cdots, U_{K}\right\}$ and $\mathcal{T}^{c}=\left\{R, U_{1}\right\}$, and applying the cut-set theorem in [11] to this cut leads to the following inequality

$$
\sum_{j=2}^{K} R_{j 1}+\sum_{j=2}^{K} R_{j c} \leq I\left(\mathbf{x}_{2}, \cdots, \mathbf{x}_{K} ; \mathbf{y}_{r} \mid \mathbf{x}_{1}\right)
$$

During the $\mathrm{BC}$ phase, the messages from $\left\{U_{2}, \cdots, U_{K}\right\}$ will be relayed to $U_{1}$, we consider $\mathcal{T}=\{R, 2, \cdots, K\}$ and $\mathcal{T}^{c}=$ $\left\{U_{1}\right\}$, we get

$$
\sum_{j=2}^{K} R_{j 1}+\sum_{j=2}^{K} R_{j c} \leq I\left(\mathbf{x}_{r} ; \mathbf{y}_{1}\right)
$$

where $I(\mathbf{x} ; \mathbf{y})$ is the mutual information between random variables $\mathbf{x}$ and $\mathbf{y}, R_{i j}$ is the maximum information transfer rate from $U_{i}$ to $U_{j}, i \neq j$, and $R_{j c}$ is the maximum information transfer rate from $U_{j}$ to the remaining $K-1$ users. Using (5) and (6), we can bound the DoF as follows

$$
\begin{aligned}
& \sum_{j=2}^{K} d_{j 1}+d_{j c} \leq \min \{N,(K-1) M\} \\
& \sum_{j=2}^{K} d_{j 1}+d_{j c} \leq \min \{N, M\}
\end{aligned}
$$

Combining (7) and (8), we get

$$
\sum_{j=2}^{K} d_{j 1}+d_{j c} \leq \min \{N, M\}
$$

Similarly, by considering the messages intended to $\left\{U_{2}, \cdots, K\right\}$, respectively, and taking the appropriate cuts during MAC and $\mathrm{BC}$ phases, we get $K-1$ equations similar to (9) but with different intended user, By adding the $K$ equations we obtain

$$
D_{t} \leq K \min \{N, M\}
$$

\section{ACHIEVABILITY}

In this section, we describe the transmission strategies that can achieve the optimal DoF mentioned in Theorem 1 We split our proof into two cases according to the relationship between $M$ and $N$. Our achievability schemes depend mainly on finding linearly independent subspaces in which each pair of users can align their bi-directional vectors. 
1) Case 1: $N<M$ : In this case, $K N$ is the dominant term in the RHS of (53). In the MAC phase, we design the beamforming matrices at the transmitting users such that the signal vector coming from each user is partially aligned at the relay with that transmitted from user 1 . We use only the common messages in our achievability proof in this case. Thus, the transmitted signals of the users in the uplink are given by

$$
\begin{aligned}
\mathbf{x}_{1} & =\sum_{j=2}^{K} \boldsymbol{V}_{1 c}^{j} \mathbf{s}_{1 c} \\
\mathbf{x}_{j} & =\boldsymbol{V}_{j c} \mathbf{s}_{j c}, \quad 2 \leq j \leq K
\end{aligned}
$$

where $\mathbf{s}_{j c}$ is $N /(K-1) \times 1$ data vector, for $j \in\{1, \cdots, K\}$, containing common messages to be delivered from user $j$ to the remaining $K-1$ users. The beamforming matrices $\boldsymbol{V}_{1 c}^{j}$ and $\boldsymbol{V}_{j c}$ of dimensions $M \times N /(K-1)$, for $j \in\{2, \cdots, K\}$, are chosen such that

$$
\mathcal{S}\left\{\boldsymbol{H}_{1 R} \boldsymbol{V}_{1 c}^{j}\right\}=\mathcal{S}\left\{\boldsymbol{H}_{j R} \boldsymbol{V}_{j c}\right\}, \quad 2 \leq j \leq K
$$

where the beamforming matrices at user 1 are chosen randomly, and the rest are chosen as follows

$$
\boldsymbol{V}_{j c}=\boldsymbol{H}_{j R}^{\dagger} \boldsymbol{H}_{1 R} \boldsymbol{V}_{1 c}^{j} .
$$

The relay will receive a superposition of the transmitted signals from all the network users. Thus, the received signal at the relay is given by

$$
\mathbf{y}_{r}=\sum_{j=2}^{K} \boldsymbol{H}_{1 R} \boldsymbol{V}_{1 c}^{j}\left(\mathbf{s}_{1 c}+\mathbf{s}_{j c}\right)+\mathbf{z}_{r} .
$$

Let $\mathbf{w}_{1 j}$ where $j \in\{2, \cdots, K\}$ be the $N /(K-1) \times 1$ vector which contains a noisy linear combination of $\mathbf{s}_{1 c}$ and $\mathbf{s}_{j c}$. The relay can obtain $\mathbf{w}_{1 j}=\boldsymbol{F}_{1 j}^{H} \mathbf{y}_{r}$, where $\boldsymbol{F}_{1 j} \in \mathbb{C}^{N \times N /(K-1)}$ is the zero-forcing matrix used by the relay to remove the undesired terms. Thus the relay obtains

$$
\mathbf{w}_{1 j}=\boldsymbol{F}_{1 j}^{H} \boldsymbol{H}_{1 R} \boldsymbol{V}_{1 c}^{2}\left(\mathbf{s}_{1 c}+\mathbf{s}_{j c}\right)+\boldsymbol{F}_{1 j}^{H} \mathbf{z}_{r} .
$$

Since all the decoded vectors at the relay contain common messages, these vectors should be transmitted to all the users in the downlink. In the broadcast phase, the relay transmits

$$
\mathbf{x}_{r}=\sum_{j=2}^{K} \boldsymbol{T}_{1 j} \mathbf{w}_{1 j}
$$

where $\boldsymbol{T}_{1 j}$ 's are randomly chosen precoding matrices, of dimensions $N \times N /(K-1)$. Now, we consider the received signal at users $j$ which is given by

$$
\mathbf{y}_{j}=\boldsymbol{H}_{R j} \sum_{i=2}^{K} \boldsymbol{T}_{1 i} \mathbf{w}_{1 i}+\mathbf{z}_{j} .
$$

We use zero-forcing at user $j$ to get $\mathbf{w}_{1 j}$, for $j \in\{2, \cdots, K\}$. For example, let $\boldsymbol{N}_{12} \in \mathbb{C}^{M \times N /(K-1)}$ be the zero-forcing matrix used by user 2 such that

$$
\boldsymbol{N}_{12} \subseteq \mathcal{N}\left\{\left(\boldsymbol{H}_{R 2} \boldsymbol{T}_{13}, \cdots, \boldsymbol{H}_{R 2} \boldsymbol{T}_{1 K}\right)^{T}\right\} .
$$

As a result, user 2 can obtain $\mathbf{w}_{12}=\boldsymbol{N}_{12}^{H} \mathbf{y}_{2}$. Similarly, user 2 can obtain $\mathbf{w}_{1 j}=\boldsymbol{N}_{1 j}^{H} \mathbf{y}_{1}$, for $j \in\{3, \cdots, K\}$. Now, user 2 can simply decode $\mathbf{s}_{1 c}$ by removing the contribution of $\mathbf{s}_{2 c}$ from $\mathbf{w}_{12}$, then decodes $\mathbf{s}_{j c}$ by subtracting $\mathbf{s}_{1 c}$ from $\mathbf{w}_{1 j}$ achieving $N$ DoF. Finally, user 1 recovers $\mathbf{s}_{j c}$ by subtracting $\mathbf{s}_{1 c}$ from $\mathbf{w}_{1 j}$, for $2 \leq j \leq K$, achieving $N$ DoF. Thus, this scheme achieves a total of $K N$ DoF which is equal to the upper bound. It should be noted that if $N$ is not divisible by $K-1$, we consider a $(K-1)$-time-slot-symbol extension of the channel [12], and then proceed with designing the transmit strategy as explained above.

2) Case 2: $N \geq M$ : In this case, $D_{t}=K M$. We shut down $N-M$ antennas at the relay and use the same achievability scheme of case 1 where each user sends $M /(K-1)$ common messages to the remaining $K-1$ users.

This completes the proof of the achievability of Theorem 1

Corollary. The total DoF of the K-user MIMO mRC with private and common messages is greater than the total DoF achievable with private messages only.

Proof: Table I] compares the total DoF of the MIMO mRC in both cases.

In fact, the limited number of antennas at the relay always represented a bottleneck in achieving higher DoF. This problem becomes more noticeable as the number of users in the network increases, especially when direct links are missing. For example, when private messages only are used [7], the respective DoF bound can be easily achieved when $K=3$. However, as the number of users increases, i.e. $K>3$, the DoF upper bound cannot be achieved using private messages only when $\frac{K}{2} \geq \frac{N}{M} \geq \frac{K^{2}-3 K+3}{K-1}$ as Table II depicts. We conjecture that the number of antennas at the relay in this region are not sufficient for achieving the $K M$ DoF bound.

On the other hand, Table I] shows that introducing the common messages enables us to achieve up to $K N$ DoF, where the upper bound can be achieved by using the common messages only. This can be attributed to the fact that each common message is directed to $K-1$ users, and hence, its contribution to the total DoF scales up with increasing the number of users in the network. Additionally, the table shows that the gain obtained due to the existence of common messages increases linearly with $K$, when $N \leq M$.

\section{CONCLUSION}

The K-user MIMO mRC with private and common messages and with equal number of antennas at the users was studied. In this network, each user aims to exchange $K$ messages with the other $K-1$ users via a common relay; $K-1$ private messages to the other users in addition to a common message to all of them. We derived cut-set bounds on the total DoF, and showed that this upper bound, $K \min \{N, M\}$, is achievable. Thus, we provided a simple way of aligning signals at the users and at the relay.

We compared between the achievable DoF obtained in our network and those obtained from the one containing private 
TABLE I

ACHIEVABLE TOTAL DoF

\begin{tabular}{|c|c|c|c|}
\hline Case & Configuration & mRC with Private messages only & mRC with Private and Common messages \\
\hline 1 & $N \leq M$ & $D_{t}=2 N$ & $D_{t}=K N$ \\
\hline 2 & $M \leq N \leq \frac{2 K^{2}-2 K}{K^{2}-K+2} M$ & $D_{t}=2 N$ & $D_{t}=K M$ \\
\hline 3 & $\frac{2 K^{2}-2 K}{K^{2}-K+2} M \leq N \leq \frac{K}{2} M$ & $D_{t}=\frac{4 K^{2}-4 K}{K^{2}-K+2} M$ & $D_{t}=K M$ \\
\hline 4 & $\frac{K}{2} M \geq N \geq \frac{K^{2}-3 K+3}{K-1} M$ & $D_{t}=\frac{K(K-1)}{K^{2}-K+2}[2 N+(4-K)] M$ & $D_{t}=K M$ \\
\hline 5 & $N \geq \frac{K^{2}-3 K+3}{K-1} M$ & $D_{t}=K M$ & $D_{t}=K M$ \\
\hline
\end{tabular}

messages only. Consequently, we showed that introducing common messages besides the private messages can lead to achieving higher DoF gain. Moreover, we showed that this gain increases linearly with the number of users in the network. Additionally, cut-set bounds of different networks can be achieved using private and common messages. An interesting direction for future study would be to investigate the total DoF of multicast networks, where each user can send common messages to a group of users in addition to private messages.

\section{REFERENCES}

[1] R. Pabst, B. Walke, D. Schultz, P. Herhold, H. Yanikomeroglu, S. Mukherjee, H. Viswanathan, M. Lott, W. Zirwas, M. Dohler, H. Aghvami, D. Falconer, and G. Fettweis, "Relay-based deployment concepts for wireless and mobile broadband radio," IEEE Communications Magazine, vol. 42, no. 9, pp. 80-89, Sept. 2004.

[2] N. Lee, J.-B. Lim, and J. Chun, "Degrees of freedom of the MIMO Y channel: Signal space alignment for network coding," IEEE Transactions on Information Theory, vol. 56, no. 7, pp. 3332-3342, July 2010.

[3] A. Chaaban, K. Ochs, and A. Sezgin, "The degrees of freedom of the MIMO Y-channel," in IEEE International Symposium on Information Theory (ISIT), Istanbul, Turkey, July 2013, pp. 1581-1585.

[4] K. Lee, N. Lee, and I. Lee, "Achievable degrees of freedom on K-user Y channels," IEEE Transactions on Wireless Communications, vol. 11, no. 3, pp. 1210-1219, Feb. 2012.

[5] Y. Tian and A. Yener, "Signal space alignment and degrees of freedom for the two-cluster multi-way relay channel," in 1st IEEE Int Conf on Comm in China (ICCC), Aug. 2012, pp. 12-17.

[6] — , "Degrees of freedom for the MIMO multi-way relay channel," in IEEE International Symposium on Information Theory (ISIT), Istanbul, Turkey, July 2013, pp. 1576-1580.

[7] K. Liu, M. Tao, and D. Yang, "Generalized signal alignment for arbitrary MIMO two-way relay channels," in Proc. of IEEE Global Telecommunication Conference, Austin, Tx, Dec. 2014, pp. 1684-1689.

[8] S. A. Jafar and S. Shamai, "Degrees of freedom region of the MIMO X channel," IEEE Transactions on Information Theory, vol. 54, no. 1, pp. 151-170, Jan. 2008.

[9] V. R. Cadambe and S. A. Jafar, "Degrees of freedom of wireless X networks," in IEEE International Symposium on Information Theory, July 2008, pp. 1268-1272.

[10] S. A. Jafar and M. J. Fakhereddin, "Degrees of freedom for the MIMO interference channel," IEEE Transactions on Information Theory, vol. 53, no. 7, pp. 2637-2642, July 2007.

[11] T. M. Cover and J. A. Thomas, Elements of information theory. John Wiley \& Sons, 2012.

[12] S. A. Jafar, "Interference alignment: A New Look at Signal Dimensions in a Communication Network," Foundations and Trends in Communications and Information Theory, vol. 7, no. 1, pp. 1-136, 2011. 